\title{
Sex-Specific Patterns of Aging in Sexual Ornaments and Gametes
}

\author{
Charlie K. Cornwallis, ${ }^{1,2, \star}$ Rebecca Dean, ${ }^{2,3}$ and Tommaso Pizzari ${ }^{2}$ \\ 1. Department of Biology, Lund University, Lund SE-223 62, Sweden; 2. Edward Grey Institute, Department of Zoology, University of \\ Oxford, South Parks Road, Oxford OX1 3PS, United Kingdom; 3. Evolution and Environment, Department of Genetics, University \\ College London, Darwin Building, Gower Street, London WC1E 6BT, United Kingdom
}

Submitted June 10, 2013; Accepted March 7, 2014; Electronically published July 30, 2014

Online enhancement: appendix. Dryad data: http://dx.doi.org/10.5061/dryad.4kg17.

ABstract: Sex differences in age-dependent mortality and reproductive success are predicted to drive the evolution of sexually dimorphic patterns of reproductive investment over life. However, this prediction has not been fully explored because it is difficult to measure primary and secondary sexual traits over the life spans of males and females. Here we studied a population of fowl, Gallus gallus, to gain longitudinal data on a sexual ornament (the comb), quantity of gametes produced, and gamete quality (sperm velocity and egg mass) of males and females. Our results reveal pronounced differences between the sexes in age-specific patterns of reproductive investment. In males, comb size decreased linearly with age, high sperm quality early in life was associated with reduced sperm quality late in life, and high sperm production was related to early death. In contrast, female comb size and egg mass were maximized at intermediate ages, and fecundity was independent of life span. Finally, the way traits were related in males did not change over life, whereas in females the association between fecundity and comb size changed from positive to negative over the lifetime of a female, indicating that aging may lead to trade-offs in investment between traits in females. These results show that males and females differ in reproductive investment with age, in terms of both the expression of individual traits and their phenotypic covariance.

Keywords: sexual selection, senescence, age, ornaments, sperm, eggs.

Theory predicts that reproductive investment to acquire sexual partners, produce gametes, and nurture young will be traded off against somatic investment and survival (Kirkwood 1977; Kirkwood and Rose 1991; Roff 1992; Stearns 1992). Natural selection is expected to optimize the trade-off between longevity and reproduction at different stages of life according to the risk of extrinsic mortality and the potential for future reproductive success (Fisher 1930; Williams 1957; Hamilton 1966). High mor-

\footnotetext{
* Corresponding author; e-mail: charlie.cornwallis@biol.lu.se.
}

Am. Nat. 2014. Vol. 184, pp. E66-E78. () 2014 by The University of Chicago. 0003-0147/2014/18403-54731\$15.00. All rights reserved. DOI: $10.1086 / 677385$ tality during early life is predicted to favor individuals that prioritize reproductive investment when young at the expense of somatic maintenance ("live fast, die young"; Kirkwood 1977; Kirkwood and Rose 1991; Roff 1992; Stearns 1992). In turn, the reduction in the strength of selection that occurs later in life as mortality decreases is expected to generate senescence: an irreversible decline in reproduction or survival with age caused by alleles that have positive fitness effects early in life but negative effects later in life (antagonistic pleiotropy) and/or the expression of deleterious mutations during older ages (mutation accumulation; Medawar 1946; Williams 1957).

Sexual dimorphism in life-history strategies and patterns of senescence can evolve in response to sex-specific selection that occurs because of differences between males and females in their reproductive success and risk of extrinsic mortality at different ages (Vinogradov 1998; Carranza and Pérez-Barbería 2007; Clutton-Brock and Isvaran 2007; Bonduriansky et al. 2008; Berg and Maklakov 2012). In addition, at a mechanistic level genetic asymmetries between the sexes may cause differences in aging. For instance, aging is expected to be faster in the heterogametic sex due to the unmasking of deleterious mutation on the $\mathrm{X}$ or Z chromosome (the unguarded $\mathrm{X}$ [or Z] hypothesis: Trivers 1985; Maklakov and Lummaa 2013). Similarly, the asymmetrical inheritance of cytoplasmic genomes, such as mitochondria, may lead to faster aging in males because near-neutral or beneficial mutations to females will persist even if detrimental to males (the mother's curse: Gemmell et al. 2004; Zeh and Zeh 2005). However, evidence of whether the sexes differ in their aging patterns has to date been equivocal, with studies showing that patterns of aging in males are similar to those in females (Charmantier et al. 2006; Galván and Møller 2008; Zajitschek et al. 2009b), faster than those in females (Promislow 1992; Loison et al. 1999; Galván and Møller 2008; Kawasaki et al. 2008; Nussey et al. 2009), and slower than those in females 
(Promislow 1992; Reid et al. 2003, 2008; Maklakov et al. 2009; Zajitschek et al. 2009a; Evans et al. 2011). Even within the same study, the sexes have been found to exhibit different patterns of aging depending on the traits that are examined (Galván and Møller 2008; Nussey et al. 2009; Evans et al. 2011).

This lack of consistency between traits and studies may be explained by a number of factors. First, age-dependent risks of mortality and reproductive success are influenced by sexual selection, which can differ in shape and strength in males and females as well as across populations and species (Svensson and Sheldon 1998; Promislow 2003; Graves 2007; Bonduriansky et al. 2008). Sexual selection is typically stronger in males, and this can increase their mortality relative to that of females (Trivers and Campbell 1972; Promislow 1992; Kruger and Nesse 2004; CluttonBrock and Isvaran 2007). For instance, intense sexual competition can lead to the evolution of weaponry and aggressive behavior that increases predation (Owen-Smith 1993). Under such circumstances, males are expected to prioritize investment in reproduction earlier in life more than females (Kirkwood 1977; Kirkwood and Rose 1991; Roff 1992; Stearns 1992). Alternatively, when reproductive success increases with age-for instance, due to female choice for older individuals or ontogenic development of sexually selected traits—males can be selected to maximize reproductive investment later in life, even if early-life mortality is high (Fisher 1930; Partridge and Barton 1996; Graves 2007). Differences in the life-history strategies of males and females across studies and species may therefore be due to variation in the relationship between reproductive success and age as well as to differences in the effect of sexual selection on the mortality risk of males and females.

Second, it is logistically difficult to quantify how aging influences investment in the different traits that determine reproductive success across entire life spans. To reproduce, individuals must gain access to sexual partners, which requires investment in secondary sexual traits, such as ornaments and armaments (Andersson 1994). Following mating, reproductive success in females is determined by investment in the number of eggs produced and the resources allocated to each egg (Sheldon 2000). Similarly in males, reproductive success is influenced by the number and fertilizing efficiency of sperm inseminated, particularly in polyandrous species (Martin et al. 1974; Birkhead et al. 1999; Gage et al. 2004: Snook 2005; Pizzari et al. 2008; Pizzari and Parker 2009). As resources are often limited, investment in one trait, such as ornaments, can come at the expense of investment in others, such as gametes (Roff 1992; Stearns 1992; Parker 1998; Preston et al. 2011). However, little is known about how aging affects the trade-offs between different sexually selected traits. The severity of such trade-offs may often differ between the sexes and change over the lifetime of individuals, due to the combined effects of sex-specific selection and senescence (Bonduriansky et al. 2008; Nussey et al. 2008). For instance, in early life, when somatic maintenance is relatively cheap, it may be possible for individuals to simultaneously invest in multiple traits, but as aging and somatic deterioration ensue, trade-offs between traits are predicted to become more pronounced (Pianka and Parker 1975; Nussey et al. 2008). As a result, reproductive success is expected to depend on the way investment in multiple traits is traded off over life, which can be difficult to measure. Consequently, direct tests of differences in investment in analogous reproductive traits in males and females over their entire life spans are scarce, particularly outside laboratory model organisms (Nussey et al. 2008).

In this study, we examined age-dependent patterns of reproductive investment in a free-ranging population of domestic fowl, Gallus gallus domesticus. This system has a number of attributes that help overcome the challenges associated with testing theory on sex differences in aging. First, sexual selection and the traits that determine reproductive success are well characterized in populations of domestic fowl and their wild ancestors, the red junglefowl, G. gallus ssp. (Pizzari et al. 2002; Collet et al. 2012). The sexes are highly dimorphic (see fig. $1 A$ ), and under natural conditions males are exposed to more intense pre- and postcopulatory sexual selection than females (Pizzari et al. 2002; Collet et al. 2012). Males and females have a sexual ornament, the comb (see fig. 1A), and its size has been shown to increase mating success in both sexes in dyadic choice trials (males: Ligon and Zwartjes 1995; Parker and Ligon 2003; females: Cornwallis and Birkhead 2007b, 2008). Furthermore, the dynamics of sperm competition are reasonably well established in this species, being strongly dependent on an interaction between the number of sperm inseminated by rival males and their swimming velocity (Martin et al. 1974; Birkhead et al. 1999; Pizzari et al. 2008). In females, reproductive success is influenced by fecundity and egg mass, which increases chick viability (Byerly 1932; Williams 1994). Second, individuals can be followed over their entire life span, and it is known that females live slightly longer than males in the study population (adult male median life span $=2 \pm 2.2$ years [interquartile range $=1-4$ ]; adult female median life span $=3 \pm 2.2$ years [interquartile range $=2-4$ years]; see figure A1 [figs. A1 and A2 are available online] for the numbers of males and females surviving to different ages in the study population). Finally, detailed longitudinal information on sexual ornaments and gametes can be obtained from males and females on a regular basis, and previous research shows that male reproductive success declines steeply with age (Dean et al. 2010). 

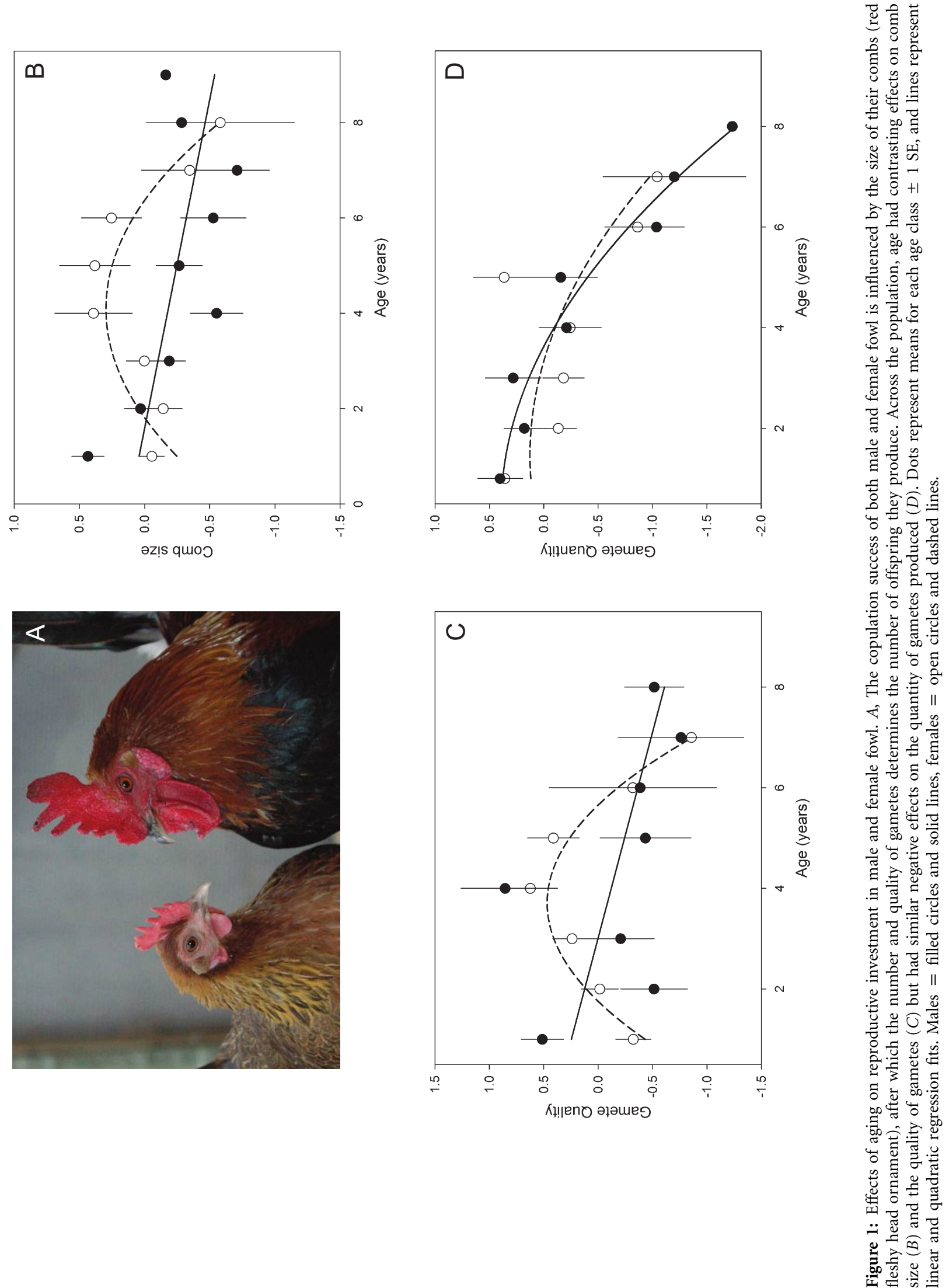
We collected longitudinal data over an 8-year period on males and females to address three key questions: (1) Do the sexes differ in their reproductive investment with age? We predict that males should prioritize investment in ornaments, gamete number, and gamete quality earlier in life than females because they live for a shorter time (Promislow 1992; Vinogradov 1998; Kruger and Nesse 2004; Clutton-Brock and Isvaran 2007; Bonduriansky et al. 2008; Zajitschek et al. 2009b), they experience more intense sexual selection, and older males have lower fertilization success (Dean et al. 2010). (2) How does lifetime reproductive investment influence longevity in males and females? We predict that within each sex higher reproductive investment will be negatively associated with longevity. Furthermore, as sexual selection is stronger in males than in females (Collet et al. 2012), we expect that the trade-off between reproductive investment and longevity will be greater in males due to higher lifetime investment in sexually selected traits. (3) Do trade-offs in the expression of different reproductive traits increase with age and differ between the sexes? We predict that trade-offs between reproductive traits will become more pronounced with age due to somatic deterioration and will be most exaggerated in the sex with the greatest decline in overall reproductive performance with age (e.g., males).

\section{Methods}

\section{Study Population}

We studied a feral population of domestic fowl that is phylogenetically, morphologically, and behaviorally similar to its wild ancestor, the red junglefowl, Gallus gallus. The study was conducted at Tovetorp Zoological Research Station, Stockholm University, Sweden, during the months of May to August from 2002 to 2009. Birds in this population are kept over winter in outdoor aviaries with access to heated indoor housing and in groups of approximately equal size and sex ratio across the population. In 2006, birds were kept indoors over the winter to increase biosecurity during the avian influenza pandemic; however, when taking into account age, sex, and their interaction, this prolonged indoor housing did not affect comb size in that year compared with other years (mean standardized comb size in $2006=0.01 \pm 0.03$; in all other years $=$ $\left.-0.07 \pm 0.09 ; F_{1,884}=0.02, P=.88\right)$. During the breeding season, birds were kept in smaller experimental groups consisting of either single-sex groups (up to 15 individuals), pairs, or small mixed-sex groups (from 2 males and 3 females up to 3 males and 6 females), and groups were occasionally allowed to range freely. Birds were rotated among experimental groups throughout the breeding season, and all ages were represented in all treatments. Birds are treated for mites and coccidiosis during outbreaks, and predation occurs, although rarely. Culling or removal of birds to manage population size occurred at the start of 2009, and no culled birds are included in this analysis. Birds in this population are individually tagged before they reach sexual maturity, so ages of all birds are known.

\section{Measuring Comb Size}

Comb size was measured using a digital photograph of the bird's head and Adobe Photoshop to calculate the comb area (Cornwallis and Birkhead 2007b). Individuals were measured repeatedly throughout the breeding season (sample sizes: $N_{\text {males }}=99, N_{\text {total observations }}=403, N_{\text {females }}=$ 118 , and $N_{\text {total observations }}=796$ ), and an average measure per individual per season was included in the analysis, resulting in the following sample sizes: $N_{\text {males }}=99$, $N_{\text {observations }}=234, N_{\text {females }}=118$, and $N_{\text {observations }}=251$.

\section{Measuring Gamete Number and Quality in Males and Females}

For males, we measured gamete number and quality by collecting ejaculates between 16:30 and 22:00, the peak time of sexual activity (Løvlie and Pizzari 2007). Males were presented with females and allowed to copulate until sexually satiated with 4 different females using a controlled copulation technique (Pizzari et al. 2003). Previous trials have shown that copulating until satiation with 4 females often results in males failing to produce ejaculates with additional females (Cornwallis and Birkhead 2007a). Therefore, it is believed that this protocol collects the vast majority of a male's sperm stores. After ejaculates were collected, sperm number was calculated using a spectrophotometer and a standard curve of the relationship between sperm number and light absorbance (Bakst and Cecil 1997). Total sperm number ejaculated was used in each analysis and was calculated as the sum of all sperm ejaculated during the sequence of copulations. Sperm quality was measured as sperm swimming speed (average path velocity in microns per second) using computerassisted sperm analysis with Sperm Class Analyzer (ver. 3.0.3) and was recorded for every third ejaculate that was collected for quantifying sperm number. Sperm velocity was recorded by diluting $1 \mu \mathrm{L}$ of semen in $50-100 \mu \mathrm{L}$ of Dulbecco's modified Eagle medium to obtain a sperm concentration of approximately $2 \times 10^{6}$. Thirty microliters of this solution was placed on a microscope slide on a heated microscope stage at $41^{\circ} \mathrm{C}$ and video recorded under dark-field optics at a magnification of $\times 200$. Median sperm velocity was calculated for each ejaculate. The mean sperm velocity of all ejaculates from that male on that day was then calculated (day mean). For the analyses, we used 
a single value for each male per year calculated as the average of all day means measured over that breeding season. Data are the same as those used in Dean et al. (2010).

For females, we measured laying rate and the quality of eggs produced. Laying rate was estimated by counting the number of eggs laid divided by the total number of days monitored during the laying period, which ranged from 10 to 60 days per breeding season. The average mass of eggs that females laid throughout the season was recorded by weighing freshly laid eggs. Females were kept in small groups of 3-6 individuals, and maternal identity of eggs was ascertained by feeding females with colored lipid dyes (Pizzari et al. 2003). Individuals were measured several times both during each breeding season and across breeding seasons (for gamete quantity, $N_{\text {males }}=41, N_{\text {total observations }}=173$, $N_{\text {females }}=84$, and $N_{\text {total observations }}=431$; for gamete quality, $N_{\text {males }}=37, N_{\text {total observations }}=112, N_{\text {females }}=79$, and $N_{\text {total observations }}=401$ ). For all analyses, an average was taken for gamete quantity and gamete quality for each individual at each age, resulting in the following sample sizes: for gamete quantity, $N_{\text {males }}=41, N_{\text {total observations }}=82, N_{\text {females }}=84$, and $N_{\text {total observations }}=142$; for gamete quality, $N_{\text {males }}=37$, $N_{\text {total observations }}=67, N_{\text {females }}=79$, and $N_{\text {total observations }}=122$ ).

\section{Statistical Analyses}

General Techniques. Prior to all analyses, continuous variables (response variables and covariates) were $Z$-transformed (mean $=0, S D=1$ ), and two-level fixed effects (e.g., sex) were converted to binary coding $(-1,1)$ so that the magnitude of parameter estimates could be compared (Gelman 2010; Schielzeth 2010). For each model, we determined the best random-effect structure by comparing corrected Akaike Information Criterion values of models with the following random effects included: (1) year + individual, (2) year + individual + individual $\times$ age, (3) year + individual + individual $\times$ age + individual $\times$ age $^{2}$, (4) year + individual $\times$ sex, (5) year + individual $\times$ sex + individual $\times$ age $\times$ sex, and (6) year + individual $\times$ sex + individual $\times$ age $\times$ sex + individual $\times$ age $^{2} \times$ sex. In all models, unstructured (co)variance matrices were fitted between individual identity and age to allow intercepts and slopes to vary independently. The random effects used in each analysis are given in tables A1A6 (tables A1-A7 are available online). The significance of the chosen random effects were tested using log-likelihood ratio tests with all fixed effects and their interactions included in the model (Self and Liang 1987). To examine the significance of fixed effects, we first tested interactions using full models with all terms included in the models and then removed higher-order interactions to test the main effects. Because data on age at last reproduction (ALR) were not available for all individuals (29 males and 41 females), we first assessed the significance of ALR and its interactions and then ran models excluding ALR to utilize all data to test the significance of other effects. The significance of fixed effects was tested using approximate $F$ tests, and degrees of freedom were calculated using the Kenward-Roger method (Kenward and Roger 1997). Parameter estimates \pm SE are presented. For interactions between covariates and fixed factors, the significance of individual slopes were tested against 0 using the $t$-test and are represented in tables by asterisks (one indicates $P<.05$, two indicates $P<.01$, and three indicates $P<.001)$. All analyses were conducted in SAS (ver. 9.2).

Specific Analyses. We performed four sets of analyses. First, we examined how comb size, gamete quality, and gamete quantity of males and females changed with age (table A1) across individuals in the population using linear mixed models (LMMs) with restricted maximum likelihood estimation (REML). We entered sex (two-level factor), age (covariate), age ${ }^{2}$, and ALR (covariate) as fixed effects. Age and age ${ }^{2}$ were fitted to test for linear and curvilinear effects of age on traits, and ALR was fitted to test whether individuals that lived different lengths of time differed in their trait expression. Interactions with sex were entered to test for differences between males and females. Year and individual identity were fitted as random effects to account for multiple observations per year and multiple observations on individuals. We also included the interaction between individual identity and age and age ${ }^{2}$ to allow for the possibility that a trait changed differentially with age across individuals (Schielzeth and Forstmeier 2009). See tables A1-A6 for specific details.

Second, we analyzed how comb size, gamete quality, and gamete quantity changed within individual males and females as they aged (response variable $=$ observed trait value at each age - mean trait value for that individual) using LMMs with REML. For these analyses, we included individuals only if we had measurements at more than two ages. We fitted sex, age, age ${ }^{2}$, and mean trait value (covariate) as fixed effects and year, individual, and interactions between individual and age and age ${ }^{2}$ as random effects. We included mean trait values for individuals in models to test whether individuals with different expression of traits (averaged over their lifetime) changed more or less with age.

Third, we tested whether the longevity (response variable: ALR) of males and females was determined by their average comb size, gamete quality, and number of gametes produced using a general linear model. For each individual for which we had data on ALR, we calculated their average comb size and the quality and quantity of gametes produced over their lifetime and included these as covariates 
in the analysis. We fitted sex and its interactions with each trait as fixed effects to test for sex-specific effects of traits on longevity.

Fourth, we analyzed how aging influenced the relationships across male and across female sexual traits (table 1), both among individuals in the population and within individuals. Across the population, we examined how the relationships between sexual traits (comb size, gamete number, and gamete quality) changed with age by fitting each trait as the response variable in a LMM and entering all other traits and their interactions with age and age ${ }^{2}$ as fixed effects. Year, individual, and the interactions between individual age and age $^{2}$ were fitted as random effects. Analyses examining changes within individuals were exactly the same, but changes in individual traits (observed trait value at each age - mean trait value for that individual) were used instead of raw phenotypic measurements.

\section{Results}

All data used to produce figures and tables for this study are deposited in the Dryad Digital Repository: http://dx .doi.org/10.5061/dryad.4kg17 (Cornwallis et al. 2014).

\section{Sex-Specific Changes in Reproductive Investment with Age}

Comb Size. Age had a pronounced effect on the comb size of males and females (table A1). However, patterns of aging in comb size were markedly different for males and females both at the level of the population ( $\operatorname{sex} \times \mathrm{age}^{2}$, $F_{1,167}=19.41, P<.0001$; table A1; fig. $\left.1 B\right)$ and within individuals $\left(\mathrm{sex} \times\right.$ age $^{2}, F_{1,44}=15.54, P=.0003$; table A2). Male comb size decreased linearly with age across the population $(\beta=-0.25 \pm 0.07$; table Al; fig. 1$)$, but there was little change within individuals $(\beta=0.16 \pm 0.12$; fig. A2; table A2), suggesting the early disappearance of more ornamented individuals. In contrast, female comb size had a quadratic relationship with age at both the population and the individual level, increasing up to a maximum at 4 years followed by a late-life decline (tables A1, A2; figs. $1 B, \mathrm{~A} 2$ ).

Gamete Quality. We found contrasting effects of age on the quality of gametes produced by males and females across the population $\left(\mathrm{sex} \times \mathrm{age}^{2}, F_{1,94}=5.12, P=.03\right.$; table A3; fig. 1C) and within individuals (sex $\times \mathrm{age}^{2}$, $F_{1,143}=13.31, P=.0004$; table A4; fig. A2). In males, there were no overall effects of age on sperm velocity, but individuals that had relatively high sperm velocity averaged across their life were much more susceptible to aging $\left(\right.$ sex $\times$ mean gamete quality $\times$ age, $F_{1,138}=13.31, P=$ .0004 ; table A4; fig. 2). Males with relatively high sperm velocity in the population maximized sperm velocity early in life at the expense of later-life sperm velocity. In contrast, females maximized the mass of their eggs at intermediate ages, and females with high average lifetime egg mass were generally more resistant to the effects of aging than other females (table A4; fig. 2).

Gamete Quantity. At the level of the population, the number of gametes males and females produced declined with age (age, $F_{1,52}=50.40, P<.0001$; table A5; fig. $1 D$ ), and this became more exaggerated later in life $\left(\right.$ age $^{2}, F_{1,139}=$ 11.82, $P=.0008$; table A5). However, within individuals the effect of age on gamete production was much weaker and linear (age, $F_{1,79}=4.49, P=.04$; table A6; fig. A2), indicating that the declines at the population level were

Table 1: Changes in relationships between traits with age in females (above the diagonal) and males (below the diagonal) across individuals in the population and within individuals

\begin{tabular}{|c|c|c|c|c|c|c|}
\hline \multirow[b]{2}{*}{ Trait, term } & \multicolumn{2}{|c|}{ Comb size } & \multicolumn{2}{|c|}{ Gamete quality } & \multicolumn{2}{|c|}{ Gamete number } \\
\hline & Population & Within individuals & Population & Within individuals & Population & Within individuals \\
\hline \multicolumn{7}{|l|}{ Comb size: } \\
\hline Main & $\ldots$ & $\ldots$ & $.19 \pm .07(.01)$ & $.20 \pm .10(.04)$ & $.18 \pm .08(.01)$ & $.20 \pm .11(.07)$ \\
\hline$\times$ age & $\ldots$ & $\ldots$ & $-.04 \pm .08(.57)$ & $-.16 \pm .14(.27)$ & $-.11 \pm .10(.25)$ & $-.12 \pm .15(.42)$ \\
\hline$\times$ age $^{2}$ & $\ldots$ & $\ldots$ & $.14 \pm .10(.18)$ & $-.06 \pm .16(.70)$ & $-.20 \pm .14(.16)$ & $-.58 \pm .21(.006)$ \\
\hline \multicolumn{7}{|c|}{ Gamete quality: } \\
\hline Main & $-.04 \pm .09(.65)$ & $-.09 \pm .12(.50)$ & $\ldots$ & $\ldots$ & $.04 \pm .10(.65)$ & $.20 \pm .11(.07)$ \\
\hline$x$ age & $.02 \pm .09(.85)$ & $.001 \pm .13(.99)$ & $\ldots$ & ... & $.26 \pm .11(.03)$ & $.22 \pm .14(.12)$ \\
\hline$\times$ age $^{2}$ & $-.17 \pm .11(.14)$ & $-.13 \pm .17(.44)$ & $\ldots$ & $\ldots$ & $.04 \pm .14(.79)$ & $.08 \pm .20(.68)$ \\
\hline \multicolumn{7}{|c|}{ Gamete number: } \\
\hline Main & $.06 \pm .10(.51)$ & $-.07 \pm .12(.55)$ & $.22 \pm .16(.17)$ & $.39 \pm .10(.0003)$ & $\ldots$ & $\ldots$ \\
\hline$\times$ age & $.02 \pm .09(.86)$ & $.08 \pm .11(.46)$ & $.18 \pm .14(.21)$ & $.14 \pm .09(.14)$ & $\ldots$ & $\ldots$ \\
\hline$\times$ age $^{2}$ & $.06 \pm .06(.31)$ & $-.03 \pm .12(.78)$ & $.05 \pm .10(.62)$ & $-.05 \pm .10(.61)$ & $\ldots$ & $\ldots$ \\
\hline
\end{tabular}

Note: Parameter estimates \pm SE are shown. Values in parentheses are $P$ values of parameter estimates tested against 0 , with italic typeface indicating significance at $P<0.05$. Significant differences between the parameter estimates for males and females at $P<0.05$ are indicated by boldface type. 


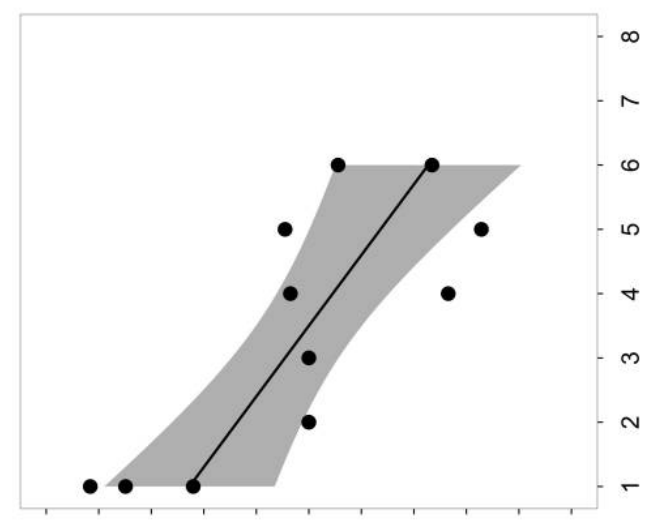

กำ

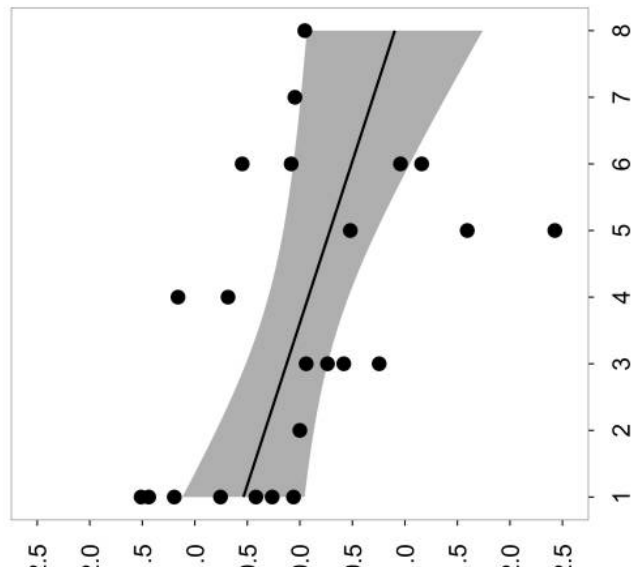

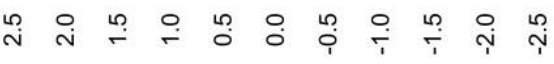

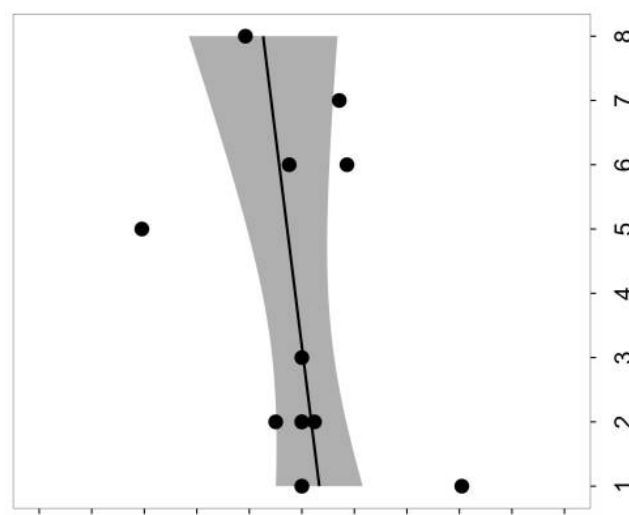

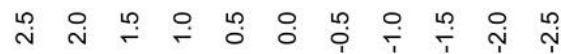

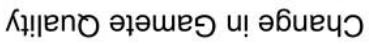
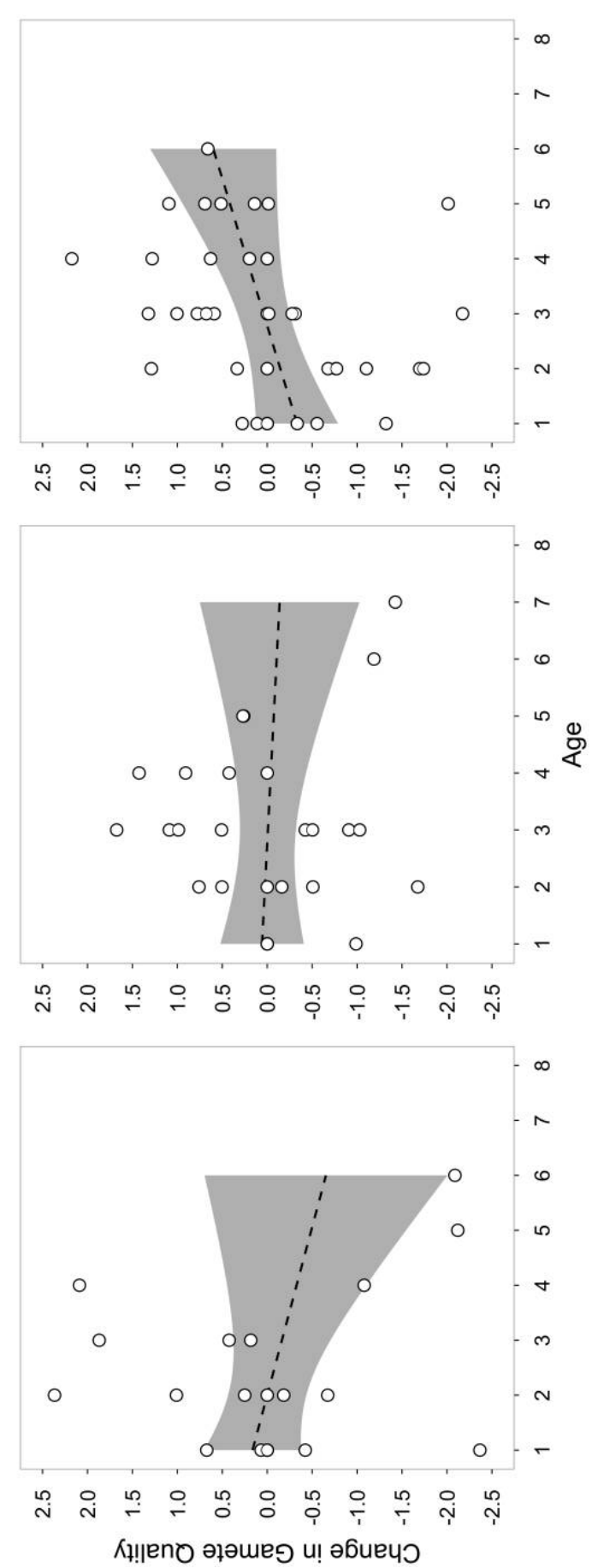

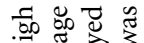

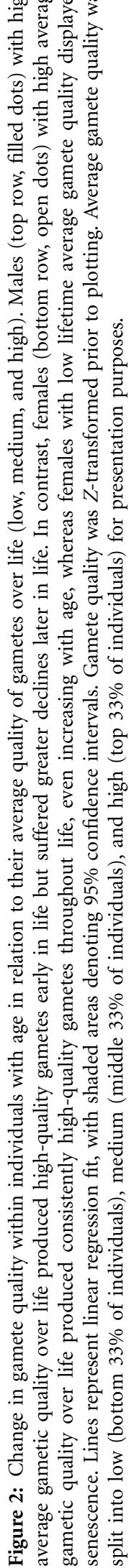


mainly due to the disappearance of individuals with high gamete production early in life.

\section{Reproductive Investment and Longevity}

Longevity was not affected by lifetime investment in comb size or gamete quality but was negatively related to the average number of gametes that individuals produced each year (gamete quantity, $F_{1,52}=5.38, P=.02$; table A7; fig. 3). Furthermore, this relationship was significant only for males, indicating that sperm production may be traded off against longevity, in contrast to females where maintaining laying rate appears to have less impact on longevity (but see below; fig. 3).

\section{Changes in Relationships between Traits with Age}

The changes in the relationships between comb size, gamete quality, and gamete quantity as individuals aged were different for males and females. In males, we found that age did not influence the relationships between any of the traits (table 1), whereas in females the relationship between comb size and gamete production changed over life. In line with previous research (Pizzari et al. 2003; Cornwallis and Birkhead 2007b; Wright et al. 2008), comb size was positively related to the number and mass of eggs produced by females at the population level (table 1). However, the association between comb size and egg number changed from positive in early life to negative in later life (fig. 4). These results indicate that as females age and somatic resources decline, trade-offs in investment between ornamental and gametic traits may become more pronounced.

\section{Discussion}

We found sexually dimorphic patterns of reproductive investment across multiple primary and secondary sexual traits. The effects of aging were generally more pronounced in males than in females. Even from very early ages, male comb size decreased and individuals that produced higherquality sperm earlier in life suffered greater declines in sperm quality later in life. In addition, high sperm production was associated with earlier death. In contrast, female comb size and egg quality first increased before declining later in life, and life span appeared independent of reproductive investment. However, we found that the relationships between traits became more negatively correlated over life in females compared with males. Together,
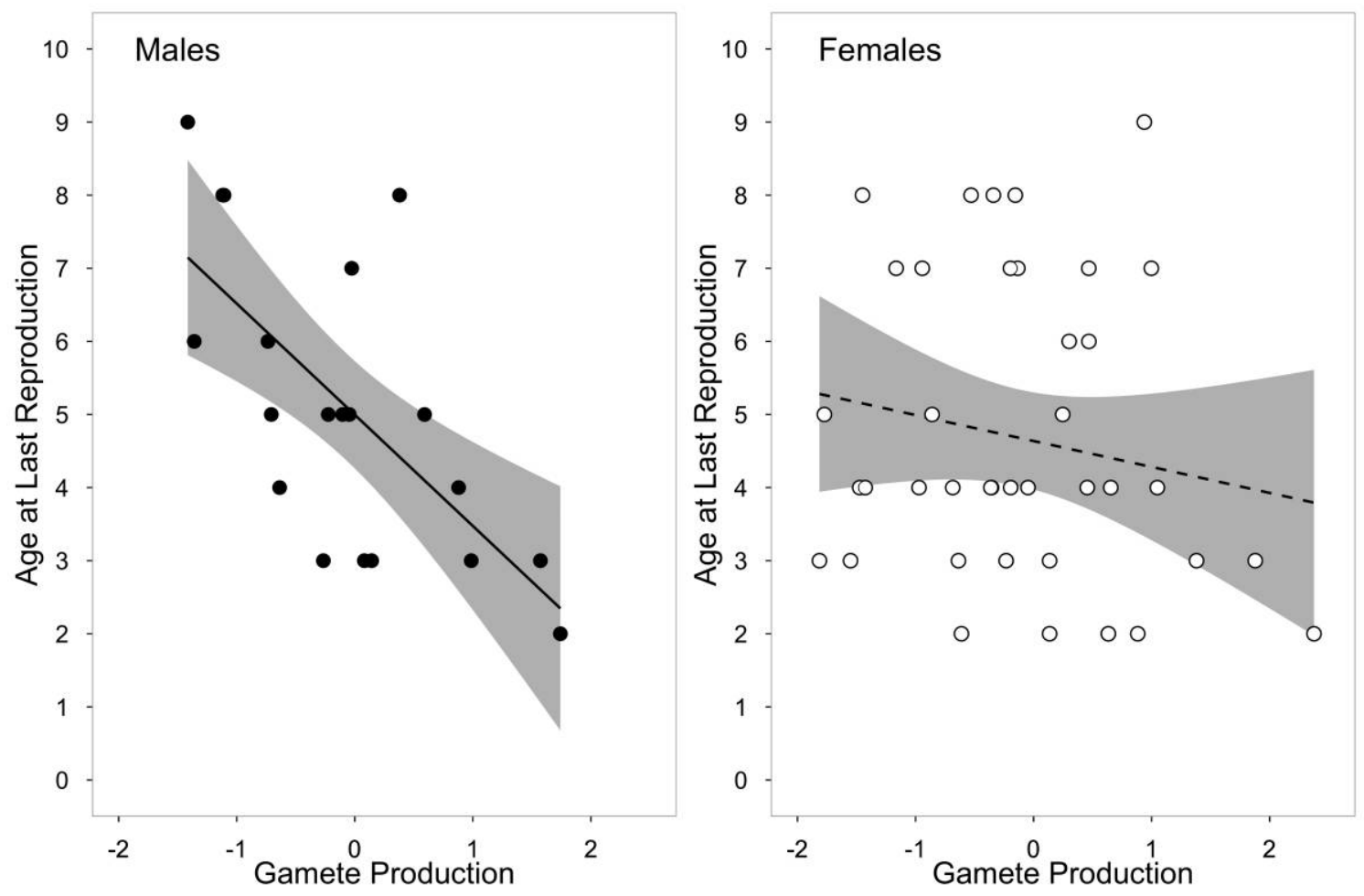

Figure 3: Relationship between longevity and gamete production in males and females. Lines represent linear regression fits, and shaded areas denote $95 \%$ confidence intervals. Gamete production was $Z$-transformed prior to plotting. 


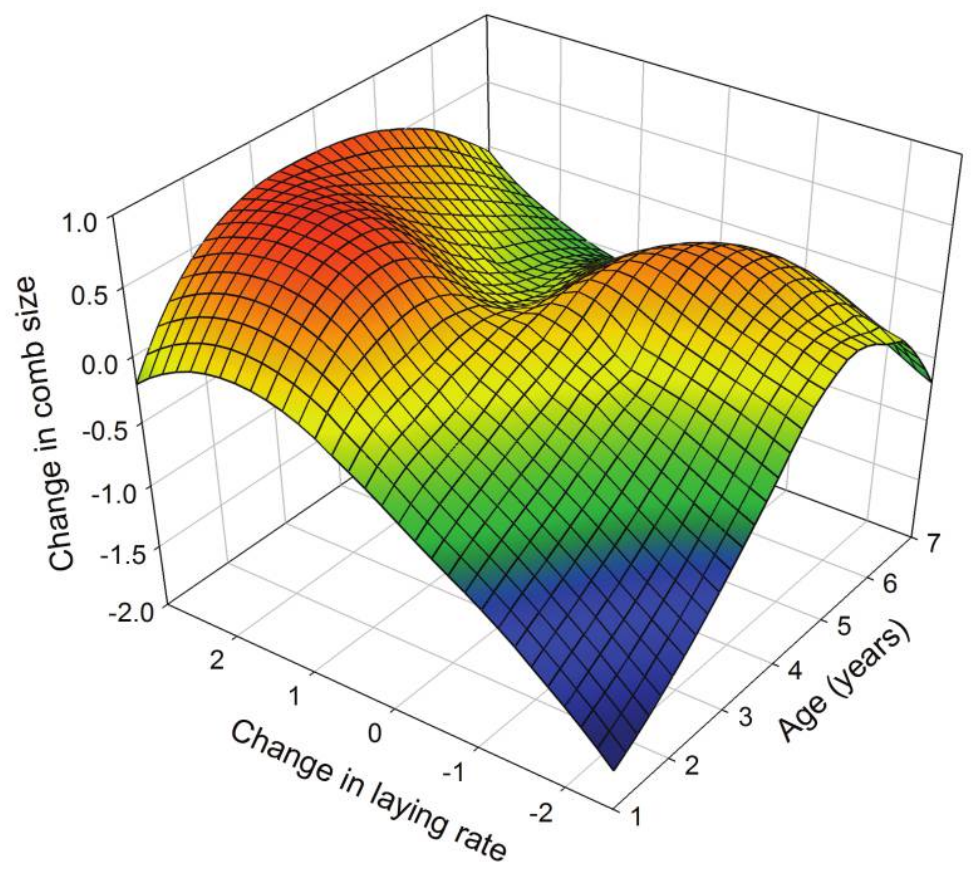

Figure 4: Change in the relationship between comb size and laying rate across age within females. The relationship is visualized using a loess smoother (100\% sampling with quadratic degree). Comb size and laying rate were $Z$-transformed prior to plotting.

these results reveal that analogous primary and secondary sexual traits in males and females change with age in different ways and that overall males appear to senesce faster than females. We discuss the implications of our results for the evolution of sex-specific life-history strategies and the maintenance of variation within populations below.

\section{Sex-Specific Life-History Strategies}

It has been proposed that differences in the strength of sexual selection and mortality rates between males and females can lead to the evolution of sex-specific reproductive strategies (Williams 1957; Trivers and Campbell 1972; Promislow 1992; Clutton-Brock and Isvaran 2007; Graves 2007; Bonduriansky et al. 2008). However, empirical findings regarding the differences in aging patterns between males and females are extremely variable between studies and across traits (e.g., Promislow 1992; Loison et al. 1999; Reid et al. 2003; Charmantier et al. 2006; Galván and Møller 2008; Reed et al. 2008; Maklakov et al. 2009; Nussey et al. 2009; Zajitschek et al. 2009a, 2009b; Evans et al. 2011), and many studies of aging have focused on a single sex (e.g., Candolin 2000a, 2000b; Jones and Elgar 2004; Wedell and Ritchie 2004; Sloter et al. 2006; Jones et al. 2007; Torres and Velando 2007; Bouwhuis et al. 2009; Gasparini et al. 2009; Dean et al. 2010; Preston et al. 2011).
Part of the problem in resolving differences between studies is that rates of aging are dependent on numerous factors, all of which can vary. First, patterns of aging are highly dependent on the traits that are examined. Even within the same study and the same sex, positive and negative relationships between trait expression and age are frequently found (e.g., Galván and Møller 2008; Nussey et al. 2009; Evans et al. 2011). Studies comparing sex differences in aging often examine different traits in males and females, making it difficult to draw conclusions about the relative rates of senescence of males and females beyond variation attributable to different traits. Second, the intensity of sexual selection on males and females can vary across species and populations, and it may increase or decrease with age (Svensson and Sheldon 1998; Promislow 2003; Graves 2007; Bonduriansky et al. 2008). Third, mortality rates for males and females can be highly variable across species, generating differences in selection for earlylife versus late-life reproductive investment (Owens et al. 2013).

In this study, we tried to resolve the above-described issues as much as possible by (1) examining a mutually expressed sexual ornament, the comb, and analogous gametic traits in males and females; (2) quantifying patterns of aging in a system where it is established that males experience stronger pre- and postcopulatory sexual selec- 
tion (Collet et al. 2012) than females (Collet et al. 2014) across their life spans; and (3) males tend to live for a shorter time (just two breeding seasons) than females. The combination of more intense sexual selection and a potentially shorter life span is currently why we think males age faster than females in this species and is in line with evidence from experimental evolution tests of aging (Maklakov et al. 2007). It is unclear at the moment what mechanisms are causing the more rapid deterioration of males versus females. However, at a genetic level it seems unlikely that the sex differences we observed are due to the asymmetrical inheritance of sex chromosomes, as this predicts faster aging in female birds (Trivers 1985; Maklakov and Lummaa 2013). It is potentially possible that mitochondrial genes play a role, as this predicts faster aging in males (Gemmell et al. 2004; Zeh and Zeh 2005). However, previous research into the genetic architecture of the comb in chickens has shown that variation in comb mass is explained by a large quantitative trait loci on chromosome 3 (Wright et al. 2008). Furthermore, there was an additional region on chromosome 1 that explained variation in female comb mass only (Wright et al. 2008). This suggests that for at least one of the traits we examined males and females have evolved somewhat independent genetic regulation of reproductive investment, and this provides the potential for responses to divergent selection pressures.

\section{Maintenance of Variation within Populations}

Explaining variation in primary and secondary sexual traits is still a major focus of sexual selection research (e.g., Kokko and Heubel 2008). A fundamental way that variation is maintained is via trade-offs in investment between traits (Roff 1992; Stearns 1992). In this study, we found that sperm production was negatively related to life span and that higher sperm quality early in life was associated with greater declines in sperm quality later in life. This contrasted with females, where there was no association between life span and any of the traits we examined. This may appear surprising, as it is commonly thought that the costs of reproduction are higher in females and so tradeoffs between traits would be more likely. There are two potential explanations that may contribute to these patterns. First, it is possible that trade-offs between reproduction and longevity in females have been removed by artificial selection for consistently high rates of egg laying. However, domestication has focused on selecting for high egg production during early life, which generates faster female life-history schedules. This is contradictory to our results that indicate that female life histories are slower than those of males, suggesting that artificial selection may have played a minor role in shaping female reproductive investment in this population. Second, trade-offs in males may be particularly pronounced because the costs of sperm production are substantial (Dewsbury 1982; Van Voorhies 1992; Pitnick 1996; Olsson et al. 1997; but see Gems and Riddle 1996). Selection for high investment in gametogenesis may be particularly strong in polyandrous species, such as the fowl, which have intense sperm competition and prolonged male mating periods due to the lack of paternal care. Consistent with this idea, in houbara bustards, Chlamydotis undulate, where sperm competition is intense and males do not provide paternal care, it was found that ejaculate quality declined dramatically with age, particularly in males that invested more in sexual displays (Preston et al. 2011). In crickets, Teleogryllus commodus, it was also found that greater investment in sexual signaling was associated with shorter life spans in males but not females (Hunt et al. 2004). However, more recent research found the opposite effect, with sexual signaling being positively associated with age (Maklakov et al. 2009), highlighting that this is an area that clearly requires further attention.

In the fowl, it is possible that the negative association between sperm production and longevity might be modulated by social status. Social dominance is strongly sexually selected in male fowl before and after mating (Collet et al. 2012), and evidence suggests that individuals may face a trade-off between investment in social dominance and sperm quality (Froman et al. 2002; Cornwallis and Birkhead 2007a; Pizzari et al. 2007). In age-structured populations, achieving dominance often requires time, favoring older males (e.g., Collis and Borgia 1992; Poston 1997; East et al. 2003). This appears to be the case for fowl populations, where older males are often dominant over younger ones (Collias and Collias 1996). Previous work on red junglefowl has shown that male social competitive ability might be more resistant to aging than aspects of reproductive performance, including mating behavior and sperm quality (Dean et al. 2010; Noguera et al. 2012). It is therefore possible that two alternative life-history strategies might be available to male fowl: one where males prioritize investment in sperm production early in life, and another where males invest in social dominance and reproductive performance later in life.

Consistent with the idea that investment in social status may be condition dependent and associated with somatic maintenance, both young and old dominant males have higher levels of plasma antioxidants than socially subordinate males in red junglefowl (Noguera et al. 2012). The relative success of these alternative strategies, however, will be dictated by rates of extrinsic mortality. When extrinsic mortality is low, investing in sperm production rather than social status may result in lower reproductive success than investing more conservatively over longer life spans. In contrast, when the risk of mortality is high, the advantage 
of early-life reproductive investment will be restored. Similar scenarios may also occur in females, as we found that higher laying rates later in life were associated with deterioration in comb size. However, the negative relationship between laying rate and comb size in females was expressed much later in life than in males, so fluctuations in mortality would have to be greater to maintain variation in females.

In summary, we show how aging affects patterns of reproductive investment in analogous primary and secondary reproductive traits in males and females. We found that early-life reproductive investment was higher in males relative to females and that this resulted in reduced longevity. We also found that in females, which appeared more robust to the effects of aging, the relationships between reproductive traits showed greater changes than males, becoming more negative with age. This suggests that the deterioration of sexually selected traits with age, as a result of early-life reproductive investment, may remove the presence of trade-offs between traits. In contrast, when decreases in somatic condition are slower, then trade-offs between reproductive traits may be more detectable. The next step will be to quantify both the physiological basis of sex differences in aging and the reproductive success of individuals adopting different reproductive strategies in relation to variation in mortality risks in wild populations.

\section{Acknowledgments}

We thank M. Baynes and H. Løvlie for help with data collection; N. Andbjer, T. Giegold, and S. Landin for animal husbandry; S. Jakobsson for hospitality and support at the Tovetorp Zoological Research Station, Stockholm University; and R. Bonduriansky, A. Crean, A. Maklakov, and two anonymous reviewers for valuable comments on the manuscript. C.K.C. was funded by an Edward Grey Institute fellowship, the Browne Fellowship from the Queen's College Oxford, and Vetenskapsrådet. R.D. was funded by a Leverhulme Award to T.P.

\section{Literature Cited}

Andersson, M. 1994. Sexual selection. Princeton University Press, Princeton, NJ.

Bakst, M., and H. Cecil. 1997. Techniques for semen evaluation, semen storage, and fertility determination. Poultry Science Association, Savoy, IL.

Berg, E. C., and A. A. Maklakov. 2012. Sexes suffer from suboptimal lifespan because of genetic conflict in a seed beetle. Proceedings of the Roval Society B: Biological Sciences 279:4296-4302.

Birkhead, T., J. Martinez, T. Burke, and D. Froman. 1999. Sperm mobility determines the outcome of sperm competition in the domestic fowl. Proceedings of the Roval Society B: Biological Sciences 266:1759-1764.

Bonduriansky, R., A. Maklakov, F. Zajitschek, and R. Brooks. 2008. Sexual selection, sexual conflict and the evolution of ageing and life span. Functional Ecology 22:443-453.

Bouwhuis, S., B. C. Sheldon, S. Verhulst, and A. Charmantier. 2009. Great tits growing old: selective disappearance and the partitioning of senescence to stages within the breeding cycle. Proceedings of the Royal Society B: Biological Sciences 276:2769-2777.

Byerly, T. 1932. Growth of the chick embryo in relation to its food supply. Journal of Experimental Biology 9:15-44.

Candolin, U. 2000a. Changes in expression and honesty of sexual signalling over the reproductive lifetime of sticklebacks. Proceedings of the Roval Society B: Biological Sciences 267:2425-2430.

. 2000b. Increased signalling effort when survival prospects decrease: male-male competition ensures honesty. Animal Behaviour 60:417-422.

Carranza, J., and F. J. Pérez-Barbería. 2007. Sexual selection and senescence: male size-dimorphic ungulates evolved relatively smaller molars than females. American Naturalist 170:370-380.

Charmantier, A., C. Perrins, R. McCleery, and B. Sheldon. 2006. Quantitative genetics of age at reproduction in wild swans: support for antagonistic pleiotropy models of senescence. Proceedings of the National Academy of Sciences of the USA 103:6587-6592.

Clutton-Brock, T. H., and K. Isvaran. 2007. Sex differences in ageing in natural populations of vertebrates. Proceedings of the Roval Society B: Biological Sciences 274:3097-3104.

Collet, J. M., R. Dean, K. Worley, D. S. Richardson, and T. Pizzari. 2014. The measure and significance of Bateman's principles. Proceedings of the Roval Society B: Biological Sciences 281:20132973.

Collet, J., D. S. Richardson, K. Worley, and T. Pizzari. 2012. Sexual selection and the differential effect of polyandry. Proceedings of the National Academv of Sciences of the USA 109:8641-8645.

Collias, N. E., and E. Collias. 1996. Social organization of a red junglefowl, Gallus gallus, population related to evolution theory. Animal Behaviour 51:1337-1354.

Collis, K., and G. Borgia. 1992. Age-related effects of testosterone, plumage, and experience on aggression and social dominance in juvenile male satin bowerbirds (Ptilonorhynchus violaceus). Auk 422-434.

Cornwallis, C. K., and T. R. Birkhead. 2007a. Changes in sperm quality and numbers in response to experimental manipulation of male social status and female attractiveness. American Naturalist 170:758-770.

- 2007b. Experimental evidence that female ornamentation increases the acquisition of sperm and signals fecundity. Proceedings of the Roval Society B: Biological Sciences 274:583-590.

2008. Plasticity in reproductive phenotypes reveals statusspecific correlations between behavioral, morphological, and physiological sexual traits. Evolution 62:1149-1161.

Cornwallis, C. K., R. Dean, and T. Pizzari. 2014. Data from: Sexspecific patterns of aging in sexual ornaments and gametes. American Naturalist, Dryad Digital Repository, http://dx.doi.org/10 $.5061 /$ dryad.4kg17.

Dean, R., C. K. Cornwallis, H. Løvlie, K. Worley, D. S. Richardson, and T. Pizzari. 2010. Male reproductive senescence causes potential for sexual conflict over mating. Current Biology 20:1192-1196.

Dewsbury, D. A. 1982. Ejaculate cost and male choice. American Naturalist 119:601-610.

East, M. L., T. Burke, K. Wilhelm, C. Greig, and H. Hofer. 2003. 
Sexual conflicts in spotted hyenas: male and female mating tactics and their reproductive outcome with respect to age, social status and tenure. Proceedings of the Roval Societv B: Biological Sciences 270:1247-1254

Evans, S. R., L. Gustafsson, and B. C. Sheldon. 2011. Divergent patterns of age-dependence in ornamental and reproductive traits in the collared flycatcher. Evolution 65:1623-1636.

Fisher, R. 1930. The genetical theory of natural selection. Oxford University Press, Oxford.

Gage, M., C. Macfarlane, S. Yeates, R. Ward, J. Searle, and G. Parker. 2004. Spermatozoal traits and sperm competition in Atlantic salmon: relative sperm velocity is the primary determinant of fertilization success. Current Biology 14:44-47.

Galván, I., and A. P. Møller. 2008. Different roles of natural and sexual selection on senescence of plumage colour in the barn swallow. Functional Ecology 23:302-309.

Gasparini, C., I. Marino, C. Boschetto, and A. Pilastro. 2009. Effect of male age on sperm traits and sperm competition success in the guppy (Poecilia reticulata). Iournal of Evolutionary Biology 23: 124-135.

Gelman, A. 2010. A question about scaling. http://andrewgelman .com/2010/04/12/a_question_abou_9/.

Gemmell, N. J., V. J. Metcalf, and F. W. Allendorf. 2004. Mother's curse: the effect of mtDNA on individual fitness and population viability. Trends in Ecology and Evolution 19:238-244.

Gems, D., and D. L. Riddle. 1996. Longevity in Caenorhabditis elegans reduced by mating but not gamete production. Nature 379:723725 .

Graves, B. M. 2007. Sexual selection effects on the evolution of senescence. Evolutionary Ecology 21:663-668.

Hamilton, W. D. 1966. The moulding of senescence by natural selection. Journal of Theoretical Biology 12:12-45.

Hunt, J., R. Brooks, M. D. Jennions, M. J. Smith, C. L. Bentsen, and L. F. Bussière. 2004. High-quality male field crickets invest heavily in sexual display but die young. Nature 432:1024-1027.

Jones, T. M., and M. A. Elgar. 2004. The role of male age, sperm age and mating history on fecundity and fertilization success in the hide beetle. Proceedings of the Roval Societv B: Biological Sciences 271:1311-1318.

Jones, T. M., R. Featherston, D. B. Paris, and M. A. Elgar. 2007. Agerelated sperm transfer and sperm competitive ability in the male hide beetle. Behavioral Ecology 18:251-258.

Kawasaki, N., C. E. Brassil, R. C. Brooks, and R. Bonduriansky. 2008. Environmental effects on the expression of life span and aging: an extreme contrast between wild and captive cohorts of Telostylinus angusticollis (Diptera: Neriidae). American Naturalist 172:346-357.

Kenward, M., and J. Roger. 1997. Small sample inference for fixed effects from restricted maximum likelihood. Biometrics 53:983997.

Kirkwood, T. B. 1977. Evolution of ageing. Nature 270:301-304.

Kirkwood, T. B., and M. R. Rose. 1991. Evolution of senescence: late survival sacrificed for reproduction. Philosophical Transactions of the Royal Society B: Biological Sciences 332:15-24.

Kokko, H., and K. Heubel. 2008. Condition-dependence, genotypeby-environment interactions and the lek paradox. Genetica 134: $55-62$.

Kruger, D. J., and R. M. Nesse. 2004. Sexual selection and the male : female mortality ratio. Evolutionary Psychology 2:66-85.

Ligon, J., and P. Zwartjes. 1995. Female red jungle fowl choose to mate with multiple males. Animal Behaviour 49:127-135.
Loison, A., M. Festa-Bianchet, J. M. Gaillard, J. T. Jorgenson, and J. M. Jullien. 1999. Age-specific survival in five populations of ungulates: evidence of senescence. Ecology 80:2539-2554.

Løvlie, H., and T. Pizzari. 2007. Sex in the morning or in the evening? females adjust daily mating patterns to the intensity of sexual harassment. American Naturalist 170:E1-E13.

Maklakov, A. A., C. Fricke, and G. Arnqvist. 2007. Sexual selection affects lifespan and aging in the seed beetle. Aging Cell 6:739-744.

Maklakov, A. A., M. D. Hall, S. J. Simpson, J. Dessmann, F. J. Clissold, F. Zajitschek, S. P. Lailvaux, et al. 2009. Sex differences in nutrientdependent reproductive ageing. Aging Cell 8:324-330.

Maklakov, A. A., and V. Lummaa. 2013. Evolution of sex differences in lifespan and aging: causes and constraints. Bioessavs 35:717724.

Martin, P., T. Reimers, J. Lodge, and P. Dziuk. 1974. The effect of ratios and numbers of spermatozoa mixed from two males on proportions of offspring. Journal of Reproduction and Fertility 39: 251-258.

Medawar, P. B. 1946. An unsolved problem in biology. Lewis, London. Noguera, J. C., R. Dean, C. Isaksson, A. Velando, and T. Pizzari. 2012. Age-specific oxidative status and the expression of pre- and postcopulatory sexually selected traits in male red junglefowl, Gallus gallus. Ecologv and Evolution 2:2155-2167.

Nussey, D. H., T. Coulson, M. Festa-Bianchet, and J. M. Gaillard. 2008. Measuring senescence in wild animal populations: towards a longitudinal approach. Functional Ecology 22:393-406.

Nussey, D. H., L. E. B. Kruuk, A. Morris, M. N. Clements, J. M. Pemberton, and T. H. Clutton-Brock. 2009. Inter- and intrasexual variation in aging patterns across reproductive traits in a wild red deer population. American Naturalist 174:342-357.

Olsson, M., T. Madsen, and R. Shine. 1997. Is sperm really so cheap? costs of reproduction in male adders, Vipera berus. Proceedings of the Roval Society B: Biological Sciences 264:455-459.

Owen, J. R., A. Scheuerlein, R. Salguero-Gomez, C. G. Camarda, R. Schaible, B. B. Casper, J. P. Dahlgren, et al. 2013. Diversity of ageing across the tree of life. Nature 505:169-173.

Owen-Smith, N. 1993. Comparative mortality rates of male and female kudus: the costs of sexual size dimorphism. Journal of Animal Ecology 62:428-440.

Parker, G. 1998. Sperm competition and the evolution of ejaculates: towards a theory base. Pages 3-54 in T. R. Birkhead and A. P. Møller, eds. Sperm competition and sexual selection. Academic Press, San Diego, CA.

Parker, T. H., and J. D. Ligon. 2003. Female mating preferences in red junglefowl: a meta-analysis. Ethology Ecology and Evolution 15:63-72.

Partridge, L., and N. H. Barton. 1996. On measuring the rate of ageing. Proceedings of the Roval Society B: Biological Sciences 263: 1365-1371.

Pianka, E. R., and W. S. Parker. 1975. Age-specific reproductive tactics. American Naturalist 109:453-464.

Pitnick, S. 1996. Investment in testes and the cost of making long sperm in Drosophila. American Naturalist 148:57-80.

Pizzari, T., C. K. Cornwallis, and D. P. Froman. 2007. Social competitiveness associated with rapid fluctuations in sperm quality in male fowl. Proceedings of the Roval Society B: Biological Sciences 274:853-860.

Pizzari, T., C. K. Cornwallis, H. Løvlie, S. Jakobsson, and T. Birkhead. 2003. Sophisticated sperm allocation in male fowl. Nature 426:7074 . 
Pizzari, T., D. P. Froman, and T. R. Birkhead. 2002. Pre- and postinsemination episodes of sexual selection in the fowl, Gallus $g$. domesticus. Heredity 88:112-116.

Pizzari, T., and G. A. Parker. 2009. Sperm competition and sperm phenotype. Pages 207-245 in T. R. Birkhead, D. J. Hosken, and S. Pitnick. Sperm biology: an evolutionary perspective. Elsevier, Amsterdam.

Pizzari, T., K. Worley, T. Burke, and D. P. Froman. 2008. Sperm competition dynamics: ejaculate fertilising efficiency changes differentially with time. BMC Evolutionary Biology 8:332.

Poston, J. P. 1997. Dominance, access to colonies, and queues for mating opportunities by male boat-tailed grackles. Behavorial Ecology and Sociobiology 41:89-98.

Preston, B. T., M. S. Jalme, Y. Hingrat, F. Lacroix, and G. Sorci. 2011. Sexually extravagant males age more rapidly. Ecology Letters 14: 1017-1024.

Promislow, D. E. 1992. Costs of sexual selection in natural populations of mammals. Proceedings of the Roval Society B: Biological Sciences 247:203-210.

. 2003. Mate choice, sexual conflict, and evolution of senescence. Behavior Genetics 33:191-201.

Reed, T. E., L. E. B. Kruuk, S. Wanless, M. Frederiksen, E. J. A. Cunningham, and M. P. Harris. 2008. Reproductive senescence in a long-lived seabird: rates of decline in late-life performance are associated with varying costs of early reproduction. American Naturalist 171:E89-E101.

Reid, J. M., E. M. Bignal, S. Bignal, D. I. McCracken, and P. Monaghan. 2003. Age-specific reproductive performance in red-billed choughs Pyrrhocorax pyrrhocorax: patterns and processes in a natural population. Lournal of Animal Ecology 72:765-776.

Roff, D. A. 1992. The evolution of life histories: theory and analysis. Chapman \& Hall, New York.

Schielzeth, H. 2010. Simple means to improve the interpretability of regression coefficients. Methods in Ecology and Evolution 1:103113.

Schielzeth, H., and W. Forstmeier. 2009. Conclusions beyond support: overconfident estimates in mixed models. Behavioral Ecology 20:416-420.

Self, S., and K. Liang. 1987. Asymptotic properties of maximum likelihood estimators and likelihood ratio tests under non-standard conditions. Journal of the American Statistical Association 82:605610.

Sheldon, B. 2000. Differential allocation: tests, mechanisms and implications. Trends in Ecology and Evolution 15:397-402.
Sloter, E., T. E. Schmid, F. Marchetti, B. Eskenazi, J. Nath, and A. J. Wyrobek. 2006. Quantitative effects of male age on sperm motion. Human Reproduction 21:2868-2875.

Snook, R. 2005. Sperm in competition: not playing by the numbers. Trends in Ecology and Evolution 20:46-53.

Stearns, S. 1992. The evolution of life histories. Oxford University Press, New York.

Svensson, E., and B. Sheldon. 1998. The social context of life history evolution. Oikos 83:466-477.

Torres, R., and A. Velando. 2007. Male reproductive senescence: the price of immune-induced oxidative damage on sexual attractiveness in the blue-footed booby. Journal of Animal Ecology 76:11611168.

Trivers, R. 1985. Social evolution. Benjamin-Cummings, Menlo Park, CA.

Trivers, R., and B. Campbell. 1972. Parental investment and sexual selection. Pages 136-179 in B. Campbell, ed. Sexual selection and the descent of man: 1871-1971. Aldine, Chicago.

Van Voorhies, W. A. 1992. Production of sperm reduces nematode lifespan. Nature 360:456-458.

Vinogradov, A. E. 1998. Male reproductive strategy and decreased longevity. Acta Biotheoretica 46:157-160.

Wedell, N., and M. G. Ritchie. 2004. Male age, mating status and nuptial gift quality in a bushcricket. Animal Behaviour 67:10591065.

Williams, G. 1957. Pleiotropy, natural selection and the evolution of senescence. Evolution 11:398-411.

Williams, T. 1994. Intraspecific variation in egg size and egg composition in birds - effects on offspring fitness. Biological Reviews of the Cambridge Philosophical Society 69:35-59.

Wright, D., S. Kerje, H. Brandstrom, K. Schutz, A. Kindmark, L. Andersson, P. Jensen, et al. 2008. The genetic architecture of a female sexual ornament. Evolution 62:86-98.

Zajitschek, F., R. Bonduriansky, S. R. K. Zajitschek, and R. C. Brooks. 2009a. Sexual dimorphism in life history: age, survival, and reproduction in male and female field crickets Teleogryllus commodus under seminatural conditions. American Naturalist 173:792-802.

Zajitschek, F., C. E. Brassil, R. Bonduriansky, and R. C. Brooks. 2009 b. Sex effects on life span and senescence in the wild when dates of birth and death are unknown. Ecology 90:1698-1707.

Zeh, J., and D. Zeh. 2005. Maternal inheritance, sexual conflict and the maladapted male. Trends in Genetics 21:281-286.

Associate Editor: Russell Bonduriansky Editor: Troy Day 